\title{
Human organic cation transporter (OCT1 and $O C T 2)$ gene polymorphisms and therapeutic effects of metformin
}

\author{
Eriko Shikata $\cdot$ Rei Yamamoto $\cdot$ Hiroshi Takane $\cdot$ \\ Chiaki Shigemasa $\cdot$ Tadasu Ikeda $\cdot$ Kenji Otsubo $\cdot$ \\ Ichiro Ieiri
}

Received: 25 August 2006/ Accepted: 27 October 2006/ Published online: 17 November 2006

(C) The Japan Society of Human Genetics and Springer 2006

\begin{abstract}
Organic cation transporters (OCTs) are responsible for the hepatic and renal transport of metformin. In this study we analyzed variants of OCT1 and OCT2 genes in 33 patients (24 responders and nine non-responders) based on the hypothesis that polymorphisms in both genes contribute to large interpatient variability in the clinical efficacy of metformin. The sequences of the 5 -flanking and coding regions of the two genes of interest were screened by singlestrand conformation polymorphism (SSCP) analysis. To compare the causative factors between responders and non-responders, we performed stepwise discriminant functional analysis. Age, body mass index (BMI) and treatment with lipid-lowering agents were demonstrated as positive predictors, and two mutations in the OCT1 gene, $-43 \mathrm{~T}>\mathrm{G}$ in intron 1 and 408Met $>$ Val $(1222 \mathrm{~A}>\mathrm{G})$ in exon 7 , were negative
\end{abstract}

E. Shikata $\cdot$ H. Takane $\cdot$ K. Otsubo $\cdot$ I. Ieiri

Department of Hospital Pharmacy, Faculty of Medicine,

Tottori University, Yonago, Japan

R. Yamamoto · C. Shigemasa

Department of Molecular Medicine and Therapeutics,

Faculty of Medicine, Tottori University, Yonago, Japan

T. Ikeda

Department of Adult and Elderly Nursing,

Faculty of Medicine, Tottori University,

Yonago, Japan

Present Address:

I. Ieiri $(\square)$

Department of Clinical Pharmacokinetics,

Graduate School of Pharmaceutical Sciences,

Kyushu University, 3-1-1, Maidashi, Higashi-ku,

Fukuoka 812-8582, Japan

e-mail: ieiri-ttr@umin.ac.jp and positive predictors, respectively, for the efficacy of metformin; the predictive accuracy was $55.5 \%$ $(P<0.05)$. Subsequent study indicated that OCT1 mRNA levels tended to be lower in human livers with the 408Met (1222A) variant, though the differences did not reach the level of significance. In this study it is suggested that $O C T 1$ and $O C T 2$ gene polymorphisms have little contribution to the clinical efficacy of metformin.

Keywords Metformin - OCT1 - OCT2 .

Polymorphisms · Pharmacokinetics .

Pharmacodynamics

\section{Introduction}

Metformin is one of the most commonly used drugs for the treatment of type 2 diabetes, but we sometimes encounter patients who do not respond sufficiently, even under approved dosage conditions (e.g., 500$750 \mathrm{mg} /$ day in Japan). Although the effects of metformin on glycemic control and lipids have been reported to be dose dependent, recent pharmacogenomic studies indicate that genetic polymorphisms of drug-metabolizing enzymes and transporters should be taken into consideration when large inter-patient variability in the intensity and duration of both drug effects and side effects is observed. Among various pharmacokinetic-related genes, since renal secretion, not hepatic metabolism, is the major route of elimination of metformin, the contribution of genetic variations in drug transporters is of interest.

Human organic cation transporters (OCTs; OCT1-3) are poly-specific transporters of small and hydrophilic 
organic cations, including toxic substances, endogenous compounds (e.g., dopamine and serotonin), and clinically used drugs (e.g., procainamide and amantadine) (Jonker and Schinkel 2004). Among the OCT family, OCT1 is expressed predominantly in the basolateral membrane of hepatocytes, and mouse Oct1, which is homologically and functionally similar to OCT1, is responsible for the hepatic uptake of metformin (Wang et al. 2002, 2003). Although the precise mechanism of the action of metformin remains unclear, it is believed that hepatic uptake is an essential step in reducing hepatic glucose production as well as the occurrence of life-threatening side effects such as lactic acidosis (Hundal et al. 2000; Stumvoll et al. 1995; Wang et al. 2002). Recently, a number of single nucleotide polymorphisms (SNPs) has been identified in the OCT1 gene. Some of these SNPs have been found to be associated with altered in vitro transport activity (Hundal et al. 2000; Sakata et al. 2003; Shu et al. 2003; Takeuchi et al. 2003).

In the kidney, OCT2, another subfamily of the OCT family, is expressed on the basolateral membrane of the proximal tubule epithelium and is involved in the uptake of many xenobiotics from the bloodstream into renal epithelial cells (Jonker and Schinkel 2004). Kimura et al. (2005) demonstrated that metformin is a good substrate for OCT2, using HEK293 cells expressing OCT2. Similar to those in the OCT1 gene, functionally different variants have been identified in the OCT2 gene (Leabman et al. 2002).

We hypothesized that large inter-patient variability in the clinical efficacy of metformin may occur as a result of variations in $O C T 1$ and/or $O C T 2$. In this report we evaluated the functional significance of genetic polymorphisms of $O C T 1$ and $O C T 2$ genes with regard to the efficacy of metformin in patients with type 2 diabetes. To date, no study has addressed the genotype-phenotype relationship in light of $O C T$ in humans.

\section{Materials and methods}

Study subjects

Thirty-three patients (nine men and 24 women; mean age 60 years, range 29-73 years) treated with metformin for at least 1 month were enrolled. We excluded patients who discontinued metformin because of adverse effects (e.g., diarrhea and headache). There are no generally accepted criteria in the clinical cut-off point to divide patients into responders and nonresponders. Thus, we selected the criteria empirically, based on our clinical experiences and a previous report (Takei et al. 2001) as follows: (1) responders $[n=24$; mean age 62 years, range $29-73$ years; mean body mass index (BMI) $25.4 \mathrm{~kg} / \mathrm{m}^{2}$, range $20.4-34.5 \mathrm{~kg} / \mathrm{m}^{2}$ ], i.e., those whose $\mathrm{HbA}_{1 \mathrm{c}}$ levels had decreased by more than $0.5 \%$ from the baseline within 3 months of metformin therapy and had remained low for more than 3 months; and (2) non-responders $(n=9$; mean age 56 years, range 34-69 years; mean BMI $25.1 \mathrm{~kg} / \mathrm{m}^{2}$, range $17.8-$ $30.6 \mathrm{~kg} / \mathrm{m}^{2}$ ), i.e., those for whom either metformin therapy had been discontinued within 3 months and/or after another hypoglycemic drug (e.g., sulfonylurea) had been added to the therapy because of insufficient improvement in $\mathrm{HbA}_{1 \mathrm{c}}$ levels. Eighteen of the responders and six of the non-responders were treated with the maximum approved daily dose in Japan (i.e., $750 \mathrm{mg} /$ day). Eight of the responders and four of the non-responders received metformin monotherapy, and others were co-medicated with sulfonylurea, $\alpha$-glycosidase inhibitor or insulin. This study was approved by the Ethics Review Board of the Faculty of Medicine, Tottori University, and all subjects gave informed consent before participating.

\section{Identification of variants in $O C T 1$ and $O C T 2$ genes}

Genomic DNA was extracted from peripheral blood. The primer design was based on the sequence of the 5 -flanking region and the intron/exon junction of $O C T 1$ and $O C T 2$ genes (GenBank accession number AL353625 for OCT1, AL162582 for OCT2). Primers were designed to divide all 11 exons of each gene into fragments of approximately $350 \mathrm{bp}$ so that mutations could be screened by subsequent single-strand conformation polymorphism (SSCP) analysis. Polymerase chain reaction (PCR) products were sequenced either directly or after subcloning on an ABI 3100 automatic sequencer (Applied Biosystems, Foster City, VA, USA).

\section{Quantitative real-time PCR}

Total RNA was extracted with an RNAeasy kit (Qiagen, Hilden, Germany) from 58 human liver samples (33 Caucasian and 25 Japanese non-diabetic donors), and reverse transcribed into cDNA using oligo dT primers and reverse transcriptase. OCT1 mRNA was quantified by real-time PCR using an ABI PRISM 7700 sequence detector (Applied Biosystems) with SYBR-green detection of reaction products. Primers for OCT1 mRNA were directed at a sequence that spans the junction of exons 9 and 10, corresponding to open reading frame $1437-1509 ; 5$-CAC 
CCCCTTCATAGTCTTCAG-3' (forward) and 5'-GCC CAACACCGCAAACAAAAT-3' (reverse). The copy number of the transcript was measured against the copy-number standard curve of cloned target templates consisting of serial tenfold dilution points. $\beta_{2}$-microgloblin mRNA was used as the reference gene for OCT1 mRNA.

\section{Statistical analysis}

The significance of differences in allelic frequency was calculated by $\chi^{2}$ analysis using $2 \times 2$ contingency tables. Statistical differences among the data for each group were determined by analysis of variance (ANOVA), followed by the Fisher least significant difference test. To compare the causative factors between responders and non-responders, we performed stepwise discriminant functional analysis. At each step, improvement in the $\chi^{2}$ and the $P$ values was used to check whether the variable entered at that step significantly improved the discrimination. The independent variables were as follows: polymorphisms, gender, age, duration of disease, types and numbers of co-medicated anti-hyperglycemic drugs, daily dose of metformin, BMI, aspartate aminotransferase, alanine aminotransferase, total cholesterol, high-density lipoprotein (HDL) and treatment with lipid-lowering agents (statins and fibrates). Data are shown as means \pm SDs. A $P$ value $<0.05$ was considered to be significant.

\section{Results}

Although the time course of change in the mean daily dose of metformin (milligrams per kilogram per day) and the initial level of $\mathrm{HbA}_{1 \mathrm{c}}$ did not differ between the two groups, the mean $\mathrm{HbA}_{1 \mathrm{c}}$ level was significantly lower in the responder group than in the non-responder group during metformin therapy (Fig. 1).

To identify polymorphisms, we performed PCRSSCP analysis of all 11 exons of the two genes of interest (OCT1 and OCT2), using DNA obtained from all patients, and the allelic frequency was compared between the responder and non-responder groups. In the OCT1 gene, 11 polymorphisms were detected by SSCP analysis and identified by subsequent sequencing; none were novel polymorphisms (Table 1). Of these, five SNPs resulted in the following amino acid substitutions: $123 \mathrm{C}>\mathrm{G}$ (41Phe $>$ Leu), 350C $>\mathrm{T}$ (117Pro $>$ Leu), $480 \mathrm{C}>\mathrm{G}(160 \mathrm{Phe}>\mathrm{Leu}), 1022 \mathrm{C}>\mathrm{T}$ (341Pro $>$ Leu), and $1222 \mathrm{~A}>\mathrm{G}(408 \mathrm{Met}>\mathrm{Val})$. Although $480 \mathrm{C}>\mathrm{G}$, $1022 \mathrm{C}>\mathrm{T}$, and $1222 \mathrm{~A}>\mathrm{G}$ variants had a relatively
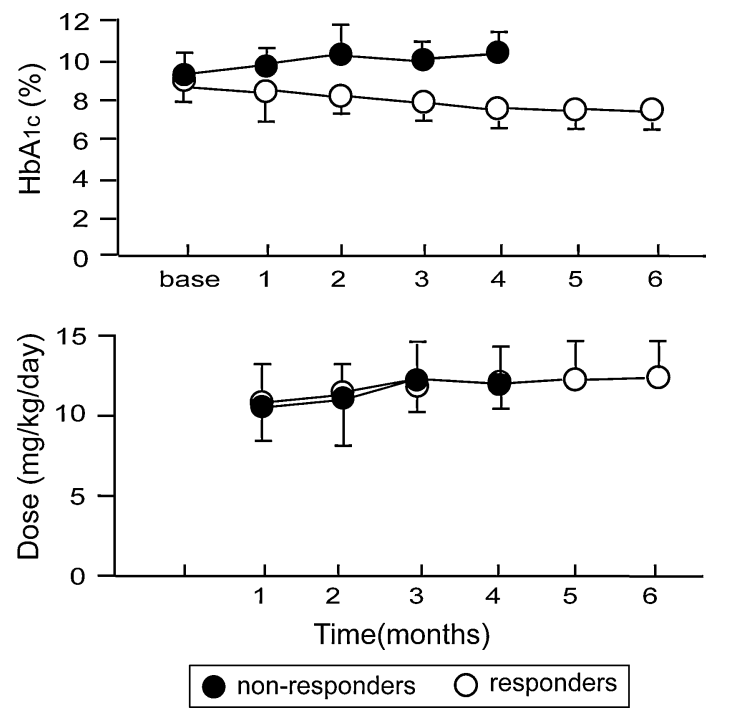

Fig. 1 Time course of changes in $\mathrm{HbA}_{1 \mathrm{c}}$ and metformin daily dose during the observation period in responders and nonresponders

high incidence, $123 \mathrm{C}>\mathrm{G}$ and $350 \mathrm{C}>\mathrm{T}$ were observed in one patient as heterozygosity. In the $O C T 2$ gene, two non-synonymous variants were observed: $602 \mathrm{C}>\mathrm{T}$ $(201 \mathrm{Thr}>\mathrm{Met})$ and $808 \mathrm{G}>\mathrm{T}(270 \mathrm{Ala}>\mathrm{Ser})$. Altogether, there were no remarkable differences in the prevalence of any mutation between responders and non-responders.

The result of discriminant functional analysis is shown in Table 2. Variables selected by the discriminant process were age, BMI, treatment with lipidlowering agents and two mutations in the OCT1 gene $(-43 \mathrm{~T}>\mathrm{G}$ and $1222 \mathrm{~A}>\mathrm{G})$. Other variables, such as duration of disease, daily dose of metformin, and types of co-medicated anti-hyperglycemic drugs, had no significant effect on the discrimination. Although age, BMI and treatment with lipid-lowering agents were demonstrated as positive predictors, $-43 \mathrm{~T}>\mathrm{G}$ and $1222 \mathrm{~A}>\mathrm{G}(408 \mathrm{Met}>\mathrm{Val})$ were negative and positive predictors, respectively, for the efficacy of metformin. Total predictive accuracy using these factors was $55.5 \%\left(\chi^{2}=5.59, P<0.05\right)$.

As shown in Table 1, since the frequency of the 408Met allele tended to be higher in non-responders than in responders ( 0.28 vs 0.19 ), and since the nonsynonymous $408 \mathrm{Met}>$ Val variant was selected as a positive predictor, we next examined the association of the $408 \mathrm{Met}>\mathrm{Val}(1222 \mathrm{~A}>\mathrm{G})$ variant with the expression of OCT1 mRNA in the human liver samples (Fig. 2). Of 58 samples, we analyzed 31 that were homozygotes for the $-43 \mathrm{~T}$ variant $(-43 \mathrm{~T} / \mathrm{T})$. The mean ( \pm SD) hepatic expression level of OCT1 in homozygotes for 408Met (1222A/1222A), heterozygotes for 
Table 1 Summary of $O C T 1$ and $O C T 2$ gene polymorphisms

\begin{tabular}{|c|c|c|c|c|c|c|c|}
\hline \multirow[t]{2}{*}{ Gene } & \multirow[t]{2}{*}{ Location } & \multirow[t]{2}{*}{ Position $^{\mathrm{a}}$} & \multirow[t]{2}{*}{ Allele $^{a}$} & \multirow{2}{*}{$\begin{array}{l}\text { Nucleotide } \\
\text { sequence }\end{array}$} & \multirow{2}{*}{$\begin{array}{l}\text { Amino acid } \\
\text { substitution }\end{array}$} & \multicolumn{2}{|c|}{ Allelic frequency $(95 \% \mathrm{CI})$} \\
\hline & & & & & & Responders $(n=24)$ & Non-responders $(n=9)$ \\
\hline \multirow[t]{22}{*}{ OCT1 } & \multirow[t]{8}{*}{ Exon 1} & \multirow[t]{2}{*}{123} & $\mathrm{C}$ & tcttCctgg & \multirow[t]{2}{*}{ 41Phe $>$ Leu } & $0.98(0.94-1.02)$ & 1.000 \\
\hline & & & G & tcttGctgg & & $0.02(-0.02-0.06)$ & 0.000 \\
\hline & & \multirow[t]{2}{*}{156} & $\mathrm{~T}$ & agagTcctg & \multirow[t]{2}{*}{ Ser52 } & $0.58(0.44-0.72)$ & $0.44(0.21-0.67)$ \\
\hline & & & $\mathrm{C}$ & agagCcctg & & $0.42(0.28-0.56)$ & $0.56(0.33-0.79)$ \\
\hline & & \multirow[t]{2}{*}{243} & $\mathrm{C}$ & cgggCgagg & \multirow[t]{2}{*}{ Gly81 } & 1.000 & $0.94(0.84-1.05)$ \\
\hline & & & $\mathrm{T}$ & cgggTgagg & & 0.000 & $0.06(-0.05-0.16)$ \\
\hline & & \multirow{2}{*}{350} & $\mathrm{C}$ & ctgcCgctg & \multirow{2}{*}{ 117Pro $>$ Leu } & 1.000 & $0.94(0.84-1.05)$ \\
\hline & & & $\mathrm{T}$ & ctgcTgctg & & 0.000 & $0.06(-0.05-0.16)$ \\
\hline & \multirow[t]{2}{*}{ Intron 1} & \multirow[t]{2}{*}{-43} & $\mathrm{~T}$ & atggTtctg & \multirow[t]{2}{*}{-} & $0.42(0.28-0.56)$ & $0.33(0.12-0.55)$ \\
\hline & & & G & atggGtctg & & $0.58(0.44-0.72)$ & $0.67(0.45-0.89)$ \\
\hline & \multirow[t]{2}{*}{ Exon 2} & \multirow[t]{2}{*}{480} & $\mathrm{C}$ & tcttCtttg & \multirow[t]{2}{*}{ 160Phe $>$ Leu } & $0.88(0.78-0.97)$ & $0.83(0.66-1.01)$ \\
\hline & & & G & tcttGtttg & & $0.13(0.03-0.22)$ & $0.17(-0.01-0.34)$ \\
\hline & \multirow[t]{2}{*}{ Exon 6} & \multirow[t]{2}{*}{1022} & $\mathrm{C}$ & $\operatorname{acgcCgcgc}$ & \multirow[t]{2}{*}{341 Pro $>$ Leu } & $0.81(0.70-0.92)$ & $0.89(0.74-1.03)$ \\
\hline & & & $\mathrm{T}$ & $\operatorname{acgcTgcgc}$ & & $0.19(0.08-0.30)$ & $0.11(-0.03-0.26)$ \\
\hline & \multirow[t]{2}{*}{ Exon 7} & \multirow[t]{2}{*}{1222} & A & ggccAtgtc & \multirow[t]{8}{*}{ 408Met $>$ Val } & $0.19(0.08-0.30)$ & $0.28(0.07-0.49)$ \\
\hline & & & G & ggccGtgtc & & $0.81(0.70-0.92)$ & $0.72(0.52-0.93)$ \\
\hline & \multirow[t]{2}{*}{ Inron 7} & \multirow[t]{2}{*}{+8} & Deletion & $($ ggtaagtt $) 0$ & & $0.81(0.70-0.92)$ & $0.72(0.52-0.93)$ \\
\hline & & & & (ggtaagtt) 1 & & $0.19(0.08-0.30)$ & $0.28(0.07-0.49)$ \\
\hline & Intron 10 & +26 & $\mathrm{C}$ & actcCgagg & & $0.98(0.94-1.02)$ & 1.000 \\
\hline & & & $\mathrm{T}$ & actcTgagg & & $0.02(-0.02-0.06)$ & 0.000 \\
\hline & & -21 & $\mathrm{C}$ & ccaaCttt & & $0.46(0.32-0.60)$ & $0.39(0.16-0.61)$ \\
\hline & & & $\mathrm{T}$ & ccaaTttt & & $0.54(0.40-0.68)$ & $0.61(0.39-0.84)$ \\
\hline OCT2 & Exon 3 & 602 & $\mathrm{C}$ & tataCgtgg & 201Thr $>$ Met & $0.98(0.94-1.02)$ & $0.94(0.84-1.05)$ \\
\hline & & & $\mathrm{T}$ & tataTgtgg & & $0.02(-0.02-0.06)$ & $0.06(-0.05-0.16)$ \\
\hline & Exon 4 & 808 & $\mathrm{G}$ & agttGctct & 270Ala > Ser & $0.92(0.88-0.96)$ & $0.94(0.84-1.05)$ \\
\hline & & & $\mathrm{T}$ & agttTctct & & $0.08(0.04-0.12)$ & $0.06(-0.05-0.16)$ \\
\hline
\end{tabular}

${ }^{a}$ Position is relative to the ATG start site, and the reference allele for each gene was obtained from the GenBank accession numbers AL353625 for OCT1 and AL162582 for OCT2

408Met $>$ Val $(1222 \mathrm{~A} / 1222 \mathrm{G})$, and homozygotes for 408Val $(1222 \mathrm{G} / 1222 \mathrm{G})$ was $0.69 \pm 0.43,0.92 \pm 0.53$, and $1.01 \pm 0.66$, respectively. Although the hepatic expression of OCT1 tended to be lower in livers with the 408Met (1222A) variant, the differences did not reach the level of significance. In the $-43 \mathrm{~T}>\mathrm{G}$ variant, the mean OCT1 expression level in $-43 \mathrm{~T} / \mathrm{T}(n=18),-$ $43 \mathrm{~T} / \mathrm{G}(n=8)$, and $-43 \mathrm{G} / \mathrm{G}(n=10)$ samples (all harbored the $1222 \mathrm{G} / 1222 \mathrm{G}$ allele) was $1.01 \pm 0.70$, $1.04 \pm 0.34$, and $1.46 \pm 0.53$, respectively.

Table 2 Stepwise discriminant functional analysis of the efficacy of metformin

\begin{tabular}{llll}
\hline Variable & Coefficient & $\chi^{2}$ value & $P$ \\
\hline Age & 0.09 & 5.59 & 0.05 \\
BMI & 0.23 & & \\
Treatment with lipid-lowering agents & 2.25 & & \\
-43T > G (intron 1) & -2.35 & & \\
408Met > Val (exon 7) & 2.51 & & \\
\hline
\end{tabular}

Predictive accuracy $=55.5 \%$

\section{Discussion}

In this study we first analyzed mutations in $O C T 1$ and $O C T 2$ and then examined the association between polymorphisms in these two genes and the efficacy of metformin, because in vitro studies have indicated that OCT1 and OCT2 are responsible, respectively, for the hepatic and renal transport of metformin (Kimura et al. 2005; Wang et al. 2002, 2003). In contrast to studies in vitro and with animals, there are no data from human studies on the contribution of these polymorphisms to the phenotypes of metformin.

In the $O C T 1$ gene, all non-synonymous variants except 41Phe $>$ Leu and 117Pro $>$ Leu have already been identified in some racial populations, with a frequency of 0.005-0.81 (Kerb et al. 2002; Shu et al. 2003). The 41Phe $>$ Leu and 117Pro $>$ Leu allele frequencies were relatively low (0.004), and they have already been reported in a Japanese population (Itoda et al. 2004). Recent expression studies have indicated that 341Pro $>$ Leu had decreased ability to transport test compounds, while 160Phe $>$ Leu and 408Met $>$ Val were unchanged (Kerb et al. 2002; Sakata et al. 2003; 


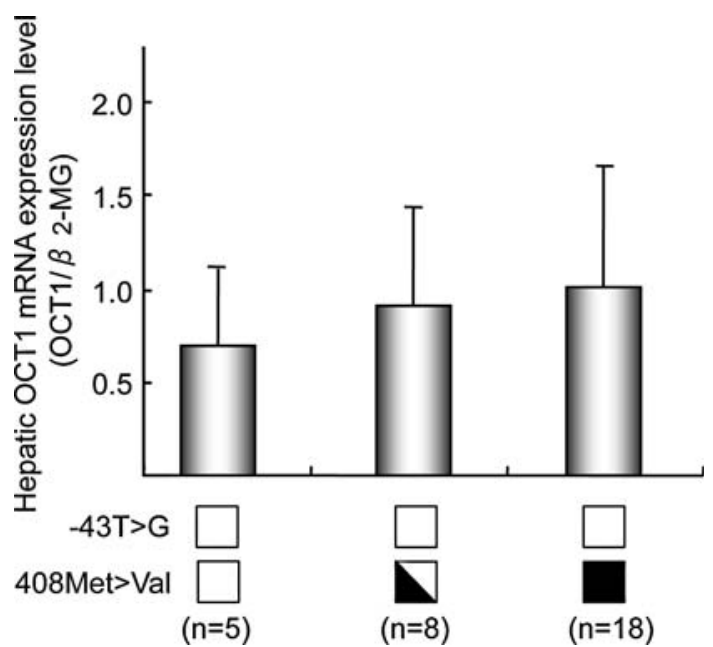

Fig. 2 Hepatic OCT1 mRNA expression levels with regard to the 408Met $>$ Val $(1222 \mathrm{~A}>\mathrm{G})$ variant. Among 58 samples, 31, which were homozygotes for the $-43 \mathrm{~T}$ variant $(-43 \mathrm{~T} / \mathrm{T})$, were analyzed. Open squares, partially filled squares and closed squares correspond to patients homozygous for the $408 \mathrm{Met}$ (1222A) allele and heterozygous and homozygous for the 408Val (1222G) allele

Shu et al. 2003). Interestingly, the 341Pro $>$ Leu variant was observed in Asian and African American populations but not in Caucasians (Shu et al. 2003); however, there was no difference in the allele frequency of 341Pro > Leu between responders and nonresponders to metformin therapy in this study.

In contrast to those in the OCT1 gene, it appears that the number of non-synonymous variants in the OCT2 gene and their allelic frequencies were lower than in other known drug transporter genes such as $M D R 1, M R P 1, M R P 2$, and $O A T P-C$ (Nishizato et al. 2003). These observations are consistent with the finding of a lower frequency of non-synonymous variants in ethnically diverse genomic DNA samples (Leabman et al. 2002). Recent population-genetic analysis has demonstrated that selection has acted against amino acid changes in OCT2 (Leabman et al. 2002), suggesting that OCT2 is relatively intolerant of non-synonymous changes. In general, the less frequent non-synonymous variants resulted in more significant and deleterious functional changes. However, the 270 Ala $>$ Ser variant was reported to exhibit subtle functional differences from the reference form of OCT2 (Leabman et al. 2002).

Although there were no remarkable differences in the prevalence of any mutation sites between responders and non-responders, we next carried out discriminant functional analysis including not only genetic polymorphisms but also the patients' background. As shown in Table 2, age, BMI and treatment with lipid-lowering agents were demonstrated as positive predictors of metformin efficacy. These observations are partially in agreement with the findings by Knowler et al. (2002), that metformin was less effective in subjects with lower BMI or a lower fasting plasma glucose concentration. BMI $>25 \mathrm{~kg} / \mathrm{m}^{2}$ is defined as obesity in Japan; $66.7 \%$ of responders and $44.4 \%$ of non-responders were obese in this study. Although the precise mechanism is unknown, these data suggest that metformin is more effective in the case of obesity-induced insulin resistance that is higher fasting plasma glucose. The contribution of lipid-lowering agents was somewhat unexpected, because metformin therapy has been reported to improve both glycemic control and lipid concentrations (i.e., plasma total and low-density lipoprotein cholesterol and triglyceride) in patients with non-insulin-dependent diabetes mellitus (DeFronzo and Goodman 1995). However, in our study, 12 responders and two non-responders were treated with lipid-lowering agents, and most of these patients (11/12 responders and 1/2 non-responders) used HMG-CoA reductase inhibitors (statins). Several studies have shown that low-density lipoprotein (LDL) size rather than plasma LDL level is more correlated with insulin resistance and eventual progression of coronary heart disease (Rizzo and Berneis 2006). Although the efficacy of modifying LDL size is different among agents (fluvastatin and atorvastatin seem to be much more effective agents than pravastatin and simvastatin), statins moderately lower all LDL subclasses, and, somehow, this process seems to make metformin more effective.

Since $-43 \mathrm{~T}>\mathrm{G}$ and $408 \mathrm{Met}>\mathrm{Val}(1222 \mathrm{~A}>\mathrm{G})$ variants were identified as negative and positive predictors, respectively, for the clinical effectiveness of metformin, we evaluated the functional significance of the latter non-synonymous variant in the expression of OCT1 mRNA, using human liver samples. Our findings indicate that samples with the 408Met (1222A) allele tended to be associated with a reduced expression level, as compared with those without the 408Met allele; however, the difference did not reach significance. A recent study using site-directed mutagenesis has indicated that point mutations in the predicted ninth transmembrane domain such as $1222 \mathrm{~A}>\mathrm{G}$ (408Met $>\mathrm{Val})$ do not lead to functional changes (Kerb et al. 2002). We also measured OCT1 mRNA expression with regard to the non-coding $43 \mathrm{~T}>\mathrm{G}$ variant; however, no significant effect was observed. In the present study, the predicted accuracy is still insufficient for its clinical application (i.e., $55.5 \%)$. Thus, if these observations are taken into consideration, the contribution of polymorphisms in 
$O C T 1$ and $O C T 2$ genes to metformin efficacy may not be as significant as our expectations had led us to believe. However, since a non-synonymous variant 408Met $>$ Val is often observed simultaneously with other non-synonymous variants (Shu et al. 2003), further in vitro and in vivo studies with regard to the haplotypic consideration, including the non-coding region, are needed to elucidate the functional properties of the variants identified in this study.

While data from only 24 responders and nine nonresponders were used, this preliminary investigation is the first study addressing the genotype-phenotype relationship of OCTs in the efficacy of metformin. However, obviously, the small number of patients is a drawback in our study. For example, co-medication of other anti-hyperglycemic drugs in both groups made it difficult for us to judge whether the decreases in $\mathrm{HbA}_{1 \mathrm{c}}$ levels in the responders are attributable to the metformin effect. Clearly, definition of the clinical cut-off point is also essential to divide patients into the two groups correctly. In order to overcome these problems, it is clear that the results in this study should be confirmed in a population study involving large numbers of patients. Nevertheless, this report provides for the possibility of OCTs' functions in humans.

Acknowledgments This work was supported by Health and Labor Sciences Research grants from the Ministry of Health, Labor, and Welfare for Research on Advanced Medical Technology. None of the authors claims any conflict of interest.

\section{References}

DeFronzo RA, Goodman AM (1995) Efficacy of metformin in patients with non-insulin-dependent diabetes mellitus. The Multicenter Metformin Study Group. N Engl J Med 333:541-549

Hundal RS, Krssak M, Dufour S, Laurent D, Lebon V, Chandramouli V, Inzucchi SE, Schumann WC, Petersen KF, Landau BR, Shulman GI (2000) Mechanism by which metformin reduces glucose production in type 2 diabetes. Diabetes 49:2063-2069

Itoda M, Saito Y, Maekawa K, Hichiya H, Komamura K, Kamakura S, Kitakaze M, Tomoike H, Ueno K, Ozawa S, Sawada J (2004) Seven novel single nucleotide polymorphisms in the human SLC22A1 gene encoding organic cation transporter 1(OCT1). Drug Metab Pharmacokinet 19:308-312

Jonker JW, Schinkel AH (2004) Pharmacological and physiological functions of the polyspecific organic cation transporters: OCT1, 2, and 3 (SLC22A1-3). J Pharmacol Exp Ther 308:2-9

Kerb R, Brinkmann U, Chatskaia N, Gorbunov D, Gorboulev V, Mornhinweg E, Keil A, Eichelbaum M, Koepsell H (2002) Identification of genetic variations of the human organic cation transporter hOCT1 and their functional consequences. Pharmacogenetics 12:591-595

Kimura N, Okuda M, Inui K (2005) Metformin transport by renal basolateral organic cation transporter hOCT2. Pharm Res 22:255-259

Knowler WC, Barrett-Connor E, Fowler SE, Hamman RF, Lachin JM, Walker EA, Nathan DM (2002) Reduction in the incidence of type 2 diabetes with lifestyle intervention or metformin. N Engl J Med 346:393-403

Leabman MK, Huang CC, Kawamoto M, Johns SJ, Stryke D, Ferrin TE, DeYoung J, Taylor T, Clark AG, Herskowitz I, Giacomini KM (2002) Pharmacogenetics of Membrane Transporters Investigators: polymorphisms in a human kidney xenobiotic transporter, OCT2, exhibit altered function. Pharmacogenetics 12:395-405

Nishizato Y, Ieiri I, Suzuki H, Kimura M, Kawabata K, Hirota T (2003) Polymorphisms of OATP-C (SLC21A6) and OAT3 (SLC22A8) genes: consequences for pravastatin pharmacokinetics. Clin Pharmacol Ther 73:554-565

Rizzo M, Berneis K (2006) The clinical relevance of low-densitylipoproteins size modulation by statins. Cardiovasc Drugs Ther 20:205-217

Sakata T, Anzai N, Shin HJ, Noshiro R, Hirata T, Yokoyama H (2003) Novel single nucleotide polymorphisms of organic cation transporter 1 (SLC22A1) affecting transport functions. Biochem Biophys Res Commun 313:789-793

Shu Y, Leabman MK, Feng B, Mangravite LM, Huang CC, Stryke D, Kawamoto M, Johns SJ, DeYoung J, Carlson E, Ferrin TE, Herskowitz I, Giacomini KM, Pharmacogenetics of Membrane Transporters Investigators (2003) Evolutionary conservation predicts function of variants of the human organic cation transporter, OCT1. Proc Natl Acad Sci U S A 100:5902-5907

Stumvoll M, Nurjhan N, Perriello G, Dailey G, Gerich JE (1995) Metabolic effects of metformin in non-insulin-dependent diabetes mellitus. N Engl J Med 333:550-554

Takei I, Miyamoto K, Funae O, Ohashi N, Meguro S, Tokui M, Saruta T (2001) Secretion of GIP in responders to acarbose in obese type2 (NIDDM) patients. J Diabetes Complications 15:245-249

Takeuchi A, Motohashi H, Okuda M, Inui K (2003) Decreased function of genetic variants, Pro283Leu and Arg287Gly, in human organic cation transporter hOCT1. Drug Metab Pharmacokinet 18:409-412

Wang DS, Jonker JW, Kato Y, Kusuhara H, Schinkel AH, Sugiyama Y (2002) Involvement of organic cation transporter 1 in the hepatic and intestinal distribution of metformin. J Pharmacol Exp Ther 302:510-515

Wang DS, Kusuhara H, Kato Y, Jonker JW, Schinkel AH, Sugiyama Y (2003) Involvement of organic cation transporter 1 in the lactic acidosis caused by metformin. Mol Pharmacol 63:1-5 